Documentation et bibliothèques

DOCUMENTATION BIBLIOTHËQUES

\title{
Vers une consolidation des services québécois d'information documentaire
}

\section{Daniel Reicher}

Volume 23, numéro 3, septembre 1977

URI : https://id.erudit.org/iderudit/1055224ar

DOI : https://doi.org/10.7202/1055224ar

Aller au sommaire du numéro

\section{Éditeur(s)}

Association pour l'avancement des sciences et des techniques de la documentation (ASTED)

\section{ISSN}

0315-2340 (imprimé)

2291-8949 (numérique)

Découvrir la revue

\section{Citer cet article}

Reicher, D. (1977). Vers une consolidation des services québécois d'information documentaire. Documentation et bibliothèques, 23(3), 123-126.

https://doi.org/10.7202/1055224ar

\section{Résumé de l'article}

Les services d'information documentaire ont trop longtemps ignoré leur importance économique et ils ont insisté uniquement sur leurs activités culturelles. Il faut convaincre le Gouvernement qu'il y va de son intérêt de consolider les services de documentation. Cet encadrement permettra de franchir les diverses étapes qui mèneront de la coopération sporadique à la rationalisation intensive dans ces domaines d'activité.
Tous droits réservés (C) Association pour l'avancement des sciences et des techniques de la documentation (ASTED), 1977
Ce document est protégé par la loi sur le droit d'auteur. L'utilisation des services d'Érudit (y compris la reproduction) est assujettie à sa politique d'utilisation que vous pouvez consulter en ligne.

https://apropos.erudit.org/fr/usagers/politique-dutilisation/ 


\title{
Vers une consolidation des services québécois d'information documentaire
}

\author{
Daniel Reicher \\ Professeur agrégé \\ École de bibliothéconomie \\ Université de Montréal \\ Président de l'ASTED, 1975-1977
}

Les services d'information documentaire ont trop longtemps ignoré leur importance économique et ils ont insisté uniquement sur leurs activités culturelles. II faut convaincre le Gouvernement qu'il y va de son intérêt de consolider les services de documentation. Cet encadrement permettra de franchir les diverses étapes qui mèneront de la coopération sporadique à la rationalisation intensive dans ces domaines d'activité.

Documentary information services, for too long, have ignored their economic importance and have insisted only on their cultural activities. We must convince the government that it is in its interest to consolidate documentary services. This framework, once established, will mean that the different steps between sporadic cooperation and intensive rationalisation in this domain will be taken.

Los servicios de información documantaria han insistido demasiado en su papel cultural dejando aparte sus funciones económicas. Hay que convencer el gobierno que tiene interés en consolidar los servicios de documentación porque una estructura gubernamental más rigida permitiria una evolución de la cooperación esporádica actual hacia una racionalización intensiva en estos campos de actividad.

Les services québécois d'information documentaire font face à une situation paradoxale: leurs ressources sont aussi éparpillées que limitées. Grand luxe pour des mal nantis! L'on pourrait évidemment argumenter que notre pauvreté documentaire tient à notre manque d'efficacité administrative, tout comme on pourrait imputer notre manque de cohésion bibliographique au défaitisme causé par cette disette de fonds.

Le cercle semble vicieux; pourtant, il n'y a aucune bonne raison pour qu'il le reste. Nous avons en main tous les renseignements nécessaires pour pouvoir planifier et justifier l'avenir de nos services. Nous savons aujourd'hui combien de lecteurs potentiels le Québec comptera en l'an 1982; ils sont déjà tous nés et leur nombre ne changera pas sensiblement à moins d'une catastrophe. Nous pouvons estimer combien d'usagers potentiels fréquenteront les universités en l'an 1995. 
Ces étudiants sont déjà tous nés... Nous pouvons aussi calculer combien de personnes âgées auront besoin d'aide documentaire spécialisée en 2037. Ces futurs sexagénaires sont en train de naître. Connaissant leur clientèle, les spécialistes en information documentaire devraient être capables de structurer leurs opérations à long terme.

Évidemment, nous admettrons sans peine que le problème est un peu moins simple que nous ne le donnons à entendre et il l'est d'autant moins que l'on ne tente même pas d'en estimer sérieusement l'ampleur. Combien de spécialistes en information documentaire, et nous insistons ici sur le terme spécialiste, savent, ne serait-ce qu'approximativement, combien de collègues partagent leur champ d'intérêt immédiat? [Y avezvous jamais songé vous-même, honorable lecteur?] Combien d'entre nous peuvent estimer, même grossièrement, la richesse bibliographique qui est amassée dans nos services d'information documentaire?

Nous connaissons notre secteur ou notre parcelle d'activité, mais nous nous préoccupons à peine de découvrir combien d'atomes crochus notre univers documentaire peut bien contenir. Cette ignorance est grave car elle nous empêche de proclamer fermement notre conviction que nous représentons un secteur économique important pour le Québec. Alors, nous nous recroquevillons dans une posture d'intellectuel perpétuellement sur la défensive parce qu'il ignore sa propre force de frappe.

Pourtant, d'après l'Annuaire du Québec de 1975/1976, nos bibliothèques publiques contenaient, en $1974,5,055,000$ volumes et fonctionnaient, grâce à 618 employés, avec un budget de $\$ 10,457,000$. Nos bibliothèques universitaires conservaient, en 1973, 9,155,000 unités documentaires et, avec 1,442 employés, dépensaient $\$ 17,698,000$ et s'attendaient, en 1974, à un budget de dix-neuf millions. Les collèges disposaient de $\$ 12,700,000$, en 1973 , pour gérer les deux millions d'unités documentaires rassemblées dans leurs bibliothèques et leurs audiovidéothèques. Nos bibliothèques scolaires rémunéraient, en 1973, 813 employés à plein temps et 2,053 à temps partiel et renfermaient plus de dix millions d'unités documentaires.

En bref, nos services d'information documentaire représentent actuellement un investissement de l'ordre du demi-milliard de dollars, dont 200 millions pour la valeur de remplacement des locaux et 300 millions pour la valeur de remplacement des collections. Nos services exigent un budget de fonctionnement annuel de l'ordre de cent millions, dont plus de $80 \%$ (la masse salariale) retourne alimenter directement l'économie québécoise.

En fait, nous nous comparons favorablement à l'industrie manufacturière du tabac avec ses quelque 4,533 employés en 1971. Nous aimons à croire que l'information scientifique et culturelle que nous fournissons vaut bien les ronds de fumée que cette industrie respectée envoie vers les nuages grâce à nos poumons. D'ailleurs, la production finale de ces usines et de nos bibliothèques paraît aboutir à des résultats similaires dans leur intangibilité.

Sans même nous donner la peine de vérifier nos intuitions, nous sommes sûr que le Gouvernement prête aux dirigeants de l'industrie 
du tabac une oreille bien moins distraite que celle tendue à l'occasion vers les spécialistes en information documentaire.

II faut noter pourtant une différence essentielle: en 1971, il y avait seize usines de tabac au Québec. Nous ne savons pas combien il y avait de services d'information documentaire à cette époque, mais nous pouvons nous souvenir que, seulement à l'Université de Montréal, il y avait plus de bibliothèques que cela. Une industrie qui se permettrait d'être aussi éparpillée aurait sans aucun doute des problèmes financiers du même ordre que les nôtres, et elle disparaîtrait rapidement.

Pourquoi nos spécialistes ne se révèlent-ils pas administrateurs plus habiles à régler ce genre de problème? De prime abord, nous pourrions admettre qu'en tant qu'intellectuels, nous ne sommes que faiblement attirés par les sciences de la gestion. Ce mea culpa fait, nous ne voyons aucune raison de laisser tomber tout le blâme sur nos épaules collectives.

Tout en admettant que nos services accusent du retard sur ceux d'autres régions ou d'autres pays, nous nous demandons comment il se fait que dans ces régions bien desservies, les services fonctionnent plus efficacement alors qu'ils sont dirigés par des spécialistes ayant la même formation que nous. Persuadé qu'avec les ressources disponibles au Québec on pourrait offrir un meilleur service, nous nous demandons si la responsabilité pour cette carence puisse être attribuable à notre ineptie politique, au sens large du mot. Ce que nous constatons avec regret, c'est que notre aptitude à convaincre les institutions dont nous relevons est très faible. Nous nous disons convaincus que le patrimoine bibliographique devrait être utilisé de façon rationnelle et rendu accessible à tous les citoyens. Cependant, nous fonctionnons comme si nous ne croyons pas en nos propres paroles. Peut-être notre comportement est-il dû à notre défaitisme, mais ce découragement, par quoi a-t-il été provoqué?

À notre avis, la situation s'explique par le fait que nos patrons (car bien peu d'entre nous fonctionnent de façon autonome) n'ont que peu d'intérêt ou de compréhension pour la coopération.

À tout seigneur, tout honneur: nous commencerons par le Gouvernement, notre patron à tous. Lorsque les spécialistes en information documentaire s'adressent au Gouvernement, à combien d'interlocuteurs aboutissent-ils?

Au ministre des Affaires culturelles, lorsqu'il s'agit de bibliothèques publiques et de certaines bibliothèques gouvernementales.

Au ministre de l'Éducation, lorsqu'il s'agit de bibliothèques scolaires, de collège et universitaires.

Au ministre des Affaires sociales pour les bibliothèques de la santé.

Au ministre des Communications, pour d'autres bibliothèques gouvernementales.

Au ministre des Travaux publics pour la plupart des locaux. 
Au ministre de l'Industrie et du Commerce pour l'industrie du livre.

La liste est longue et pourtant nous sommes persuadé d'avoir omis quelques sommités. D'ailleurs, ce polycéphalisme a fini par nous laisser à peu près indifférent car nous avons subi les effets de trop d'initiatives fragmentaires, souvent empreintes d'un zèle respectable, mais aussi d'une ardeur insuffisamment mûrie. Notons qu'à un niveau inférieur d'autorité, l'éparpillement des autorités qui nous contrôlent est encore plus accentué, plus direct et, par conséquent, encore et toujours exaspérant.

Comble d'ironie, il nous arrive plus souvent qu'à notre tour de nous faire accuser d'immobilisme comme si c'était le rôle des spécialistes en information documentaire de mettre de l'ordre dans les structures hiérarchiques qui les dominent. D'une certaine manière, c'est rendre un hommage involontaire à nos talents de classificateurs; très involontaire évidemment, quand on songe à toutes les occasions où nous nous faisons accuser de soigner notre documentation mieux que nos clients. Or, nous savons pertinemment bien que les vraies décisions ne se prennent pas à notre niveau. Nous nous heurtons à un plafond d'autorité que nous ne pouvons absolument pas franchir. Pour cette raison, il nous semble malheureusement clair que les spécialistes en information documentaire ne pourront pas régler, par eux-mêmes, le problème du cloisonnement documentaire qui les affecte. Nous avons présenté trop de mémoires et trop de rapports pour pouvoir encore rêver de faire triompher notre point de vue. À notre avis, nous devrons nous résigner à l'idée que la consolidation viendra du sommet ou qu'elle ne se fera pas.

Nous en appellons à l'intervention étatique sans gaieté de cœur, mais nous la préférons à la faillite de nos services par manque de moyens ou d'intérêt de la part des usagers.

Quelle forme devrait prendre cette intervention? Idéalement, elle serait globale pour éliminer toute incompatibilité entre les diverses unités participant à cette structure. Cependant, et sans vouloir faire montre d'un scepticisme désabusé, nous aimerions mieux voir des structures fragmentaires regroupant des services documentaires de même calibre avec un encadrement étatique complètement unifié. Évidemment, rien d'administrativement original là-dedans, mais le moment semble propice car on peut espérer un changement d'attitude de l'État envers les services d'information documentaire à cause de son empathie envers les besoins intellectuels contemporains québécois.

L'idée n'effleure même pas notre esprit que le Gouvernement n'accordera pas à nos problèmes l'attention qu'ils méritent. Ce que nous nous permettons pourtant de souligner, c'est que la simplification comptable est rarement de mise quand on discute d'activités intellectuelles.

Les membres de l'ASTED ont voulu que le congrès de 1977 concrétise les préoccupations du congrès de 1976. Le pas le plus concret que les spécialistes en information documentaire pourraient franchir dans le domaine de la rationalisation serait de convaincre le Gouvernement de se mêler carrément de nos affaires. 